Mots. Les langages du politique

Trente ans d'étude des langages du politique

(1980-2010)

\title{
L'analyse du discours des organisations internationales. Un vaste champ encore peu exploré
}

Corinne Gobin et Jean-Claude Deroubaix

\section{(Q) OpenEdition}

Journals

Édition électronique

URL : https://journals.openedition.org/mots/19872

DOI : $10.4000 /$ mots. 19872

ISSN : 1960-6001

Éditeur

ENS Éditions

Édition imprimée

Date de publication : 30 novembre 2010

Pagination : 107-114

ISBN : 978-2-84788-235-3

ISSN : 0243-6450

\section{Référence électronique}

Corinne Gobin et Jean-Claude Deroubaix, «L'analyse du discours des organisations internationales.

Un vaste champ encore peu exploré », Mots. Les langages du politique [En ligne], 94 | 2010, mis en ligne le 06 novembre 2012, consulté le 28 avril 2022. URL : http://journals.openedition.org/mots/19872 :

DOI : https://doi.org/10.4000/mots. 19872 


\section{Mots \\ Les langages du politique}

№ 94 novembre 2010

\section{Trente ans d'étude des langages du politique (1980-2010)}

ouvrage coordonné par Paul BACOT, Marlène Coulomb-GulLY, Jean-Paul Honoré, Christian Le BART, Claire Oger, Christian Plantin

\section{SOMMAIRE}

Paul Bacot, Marlène Coulomb-Gully, Jean-Paul Honoré, Christian Le Bart, Claire Oger, Christian Plantin

Le discours politique n'est pas transparent. Permanence

et transformations d'un objet de recherche

OUTILS ET ENJEUX DU DISCOURS POLITIQUE

Ruth Amossy, Roselyne Koren

Argumentation et discours politique

Christian Plantin

Argumentation-rhétorique. Les eaux mêlées

Caroline Ollivier-Yaniv

Discours politiques, propagande, communication, manipulation 
Marc Bonhomme

La caricature politique

Paul Bacot

Développement et diversification d'une onomastique politique

Ruth Wodak

The Discursive Construction of History. Brief Considerations

Henri Boyer

Les politiques linguistiques

\section{LIEUX DE LA PRODUCTION DU DISCOURS POLITIQUE}

Christian Le Bart

Parler en politique

Dominique Maingueneau

Le discours politique et son «environnement»

Alice Krieg-Planque, Claire Oger

Discours institutionnels. Perspectives pour les sciences

de la communication

Sophie Béroud, Josette Lefèvre

Le corpus syndical. Une expérience au long cours

Corinne Gobin, Jean-Claude Deroubaix

L'analyse du discours des organisations internationales.

Un vaste champ encore peu exploré

François de la Bretèque

Le retour de la parole politique dans le cinéma français

Jean-Claude Soulages

Vie et mort du citoyen cathodique

Jacques Guilhaumou

Les discours de la Révolution française.

Aperçu d'ensemble d'un trajet de recherche (1980-2009) 


\section{DISCIPLINES ET CHAMPS DE RECHERCHE POUR L'ÉTUDE DES LANGAGES DU POLITIQUE}

Philippe Braud

L'apport de la science politique à l'étude des langages du politique

Claire Blandin

L'apport de l'histoire des médias à l'étude des langages du politique

Jean-François Tétu, Bernard Lamizet

Les SIC et les langages du politique

Sylvianne Rémi-Giraud

Sémantique lexicale et langages du politique.

Le paradoxe d'un mariage difficile?

Marlène Coulomb-Gully, Juliette Rennes

Genre, politique et analyse du discours.

Une tradition épistémologique française gender blind

Johannes Angermüller

Analyser le discours politique en Allemagne (1980-2010)

Érik Neveu

L'apport de Pierre Bourdieu à l'analyse du discours.

D'un cadre théorique à des recherches empiriques

Roselyne Ringoot

Questionner le discours avec Michel Foucault.

Actualisations théoriques et actualité éditoriale

\section{ENTRETIEN}

Maurice Tournier

Mots et politique, avant et autour de 1980

La revue Mots. Les langages du politique encourage l'usage des rectifications de l'orthographe proposées par le Conseil supérieur de la langue française et approuvées par l’Académie (Journal officiel, $n^{\circ}$ 100, 6 décembre 1990). 


\section{Corinne Gobin \\ Jean-Claude Deroubaix}

\section{L'analyse du discours \\ des organisations internationales. Un vaste champ encore peu exploré}

Les organisations internationales font partie de ces appareils politico-administratifs qui ont l'habitude de produire une pléthore de textes. La référence la plus directement associée à cette notion d'organisation internationale renvoie à la réalité institutionnelle du système onusien, dont le soleil fut longtemps l'assemblée générale de l'ONU entourée de ses nombreuses agences mondiales satellites. Dans ce cas, les corpus de textes qu'il est possible de rassembler peuvent chronologiquement couvrir plus d'un demi-siècle. Ces textes, appartenant à la catégorie des discours institutionnels (voir Alice Krieg-Planque et Claire Oger dans ce volume), forment un quasi-idéal type du discours politique: fortement contraints par un rituel de production connu, ils présentent une régularité de forme et de rythme d'apparition qui facilite mise en corpus et travail de comparaison diachronique. Ces grands corpus chronologiques (Deroubaix, 2005) de discours institutionnels sont d'excellents matériaux pour aborder ce que la notion de «transformation sociale » signifie : comment varient dans le temps le contenu des «priorités de l'heure » des agendas politiques, et de façon plus générale ce qu'est "gouverner»? Cependant, force est de constater que, par rapport aux possibilités offertes, peu de chantiers scientifiques d'analyses de discours d'organisations internationales ont été à ce jour ouverts.

De nombreuses raisons peuvent être avancées : la science politique s'est longtemps focalisée sur l'étude des institutions nationales et de l'État, ce qui, dans le champ des relations internationales, faisait passer au second plan l'étude des textes des organisations internationales, pensés comme secondaires par rapport aux positions étatiques; une analyse de type juridique, sensible plutôtà l'analyse de "textes événementiels », abordés au cas par cas, a longtemps primé dans ce champ; l'abondance des textes, qui rend la sélection et la collecte difficiles, jointe au sentiment qu'il s'agit de «documents peu contraignants», ainsi

Université libre de Bruxelles, FNRS, GRAID

cogobin@ulb.ac.be

Université Paris 3, Syled

deroubaix@swing.be 
que le «style administratif» de l'écriture, assez soporifique et joliment qualifié de «style formulaire » par Gilbert Rist (2002), ont pu en décourager plus d'un...

Cependant, dans le contexte de l'internationalisation des politiques de libre-échange et des multiples travaux portant sur la «mondialisation » et/ou "globalisation ", l'importance politique croissante d'organismes comme la Banque mondiale, le Fonds monétaire international, l'Organisation mondiale du commerce..., n'a pu qu'être soulignée. Cette montée en puissance de la mise en «agenda international » des questions et de la décision politiques hors du cadre national a été particulièrement visible avec la création de l'Union économique et monétaire européenne, ses textes décisionnels devenant d'autant plus importants à analyser que la hiérarchie des sources juridiques place le droit communautaire au sommet de la pyramide de l'ordre juridique des États membres de l'UE.

Cette nouvelle configuration des lieux de pouvoir n'a pas pour autant été suivie d'une expansion de travaux spécialisés dans l'analyse des discours des institutions sociopolitiques internationales, multilatérales ou transnationales. Peut-être parce que ce champ a d'abord été très fortement investi par des analyses de type militant ${ }^{1}$ ?

Quoi qu'il en soit, un survol des acquis analytiques permet de classer en trois grands groupes les travaux scientifiques existants : les analyses quantitatives (lexicométrie), les analyses portant sur la rhétorique, les analyses de contenu thématiques ou lexicales.

\section{Les analyses lexicométriques}

Un chantier vaste et prometteur avait été, il y a quelques années, inauguré par une équipe dirigée par Jules Duchastel ${ }^{2}$, collectant des corpus issus des organisations internationales en vue d'un traitement diachronique de statistique lexicale. Malheureusement, seuls quelques travaux de recherche de fin d'études ont abouti, comme le mémoire d'Yves Proulx (2001) consacré à l'étude du discours de l'OCDE sur la transformation des politiques sociales destinées aux sans-emploi. L'analyse du vocabulaire de ces discours montre la mise en péril du pouvoir des États à gérer leurs propres politiques par la confiscation de la représentation du réel social et politique au profit d'instances d' "expertise».

Au GRAID3, à l'université libre de Bruxelles, une attention marquée a été portée aux textes produits par l'Union européenne ou par des institutions gra-

1. Rappelons que le mouvement Attac est né de la découverte d'un texte international, au début de 1998, l'accord multilatéral sur l'investissement, préparé et négocié de façon discrète par les États au sein de l'OCDE.

2. Professeur au département de sociologie de l'université du Québec à Montréal.

3. Groupe de recherche sur les acteurs internationaux et leurs discours, sous la direction de Corinne Gobin. 
vitant dans cette aire de pouvoir. On peut distinguer des travaux thématiques (analyses de politiques sociales) et des travaux cherchant à appréhender l'ensemble de la dynamique discursive d'une institution. Ainsi, à côté de l'examen des caractéristiques de statistique lexicale des mots relatifs à l'emploi et à l'organisation du travail, au développement des droits à la sécurité sociale (Gobin et al., 2005b) ou à la politique européenne de l'enseignement (Cussó, 2008), associé à une analyse de contenu par les concordances, des travaux ont porté sur la parole officielle de la Commission européenne dans ses discours d'investiture (Deroubaix, Gobin, 1994), de la Banque centrale européenne dans ses communications mensuelles (Dufresne, 2004), de la Confédération européenne des syndicats dans ses textes de Congrès (Gobin, 1993 et 2003). Ces travaux montrent l'importante césure lexicale introduite par la mise en place du projet de marché intérieur (création de «formules mobilisatrices», apparition d'un lexique neuf, transformation de l'usage de mots anciens...), le rétrécissement de l'« autonomie sémantique » des thèmes sociaux face à l'hégémonie d'un vocabulaire mercantile, l'important travail qui s'opère pour modifier le sens de ce qu'est un "pouvoir public », une adhésion forte à ce nouveau lexique par d'autres acteurs européens. Dans le corpus de textes économiques de l'UE (grandes orientations de politiques économiques), on relève que ce ne sont pas des termes économiques et/ou monétaires qui occupent la place des plus hautes fréquences, mais ceux relatifs aux questions liées à l'organisation du travail et des salaires.

Ces espaces institutionnels transnationaux ne sont pas vides et détachés des autres lieux de pouvoir; des acteurs divers gravitent dans ces espaces et naviguent d'un lieu de pouvoir à l'autre dans un cadre professionnel. L'impact de cette circulation dans des logiques institutionnelles distinctes peut dès lors être appréhendé à partir d'interviews de ces acteurs (Wagner, 2008).

\section{Les analyses portant sur la rhétorique}

Des chercheurs se revendiquant de l'analyse critique de discours et désirant la présenter comme « école » pragmatique basée sur une démarche inter- et transdisciplinaire, Norman Fairclough et Ruth Wodak, se sont également intéressés aux organisations internationales. Se nourrissant d'un travail de recontextualisation des discours, cette approche est centrée sur l'analyse des enchaînements de segments de textes et sur les effets argumentatifs, qu'ils soient logiques, rhétoriques ou à la recherche d'un consensus. L'étude des textes s'inscrit dès lors dans une conception du discours institutionnel comme pratique sociale. Ce courant s'intéresse particulièrement aux discours institutionnels de l'UE. Ainsi, Fairclough (2005) étudie-t-il la manière dont l'argumentation sur l'émergence de la société de l'information contenue dans la Déclaration 
du sommet de Lisbonne (2000) est transformée en affirmation sur l'existence en acte d'une telle société dans le Plan national stratégique roumain. II met l'accent sur la circulation de "discours nodaux» et surtout sur leur recontextualisation. Le discours programmatique international devient discours national légitime et légitimant. S'agissant de l'éducation, Susan L. Robertson (2005) montre, en comparant les discours de l'OCDE, de la Banque mondiale et du gouvernement britannique que, malgré des différences dans le projet pour l'éducation future, le soubassement idéologique des trois approches reste semblable : une approche romantique de la société basée sur l'individualisme et l'enseignement individualisé.

Sans s'inscrire dans un travail de théorisation aussi explicite que les travaux de Norman Fairclough et Ruth Wodak, le dossier dirigé par Gilbert Rist, Les mots du pouvoir. Sens et non-sens de la rhétorique internationale, est un survol multidisciplinaire très complet des caractéristiques des rapports des organisations du système onusien : rhétorique creuse, vide de pensée, asphyxiée de formules toutes faites, associée à une langue de coton en vue de susciter une adhésion universelle... et proposant comme seule voie le programme de la mondialisation mise en scène comme processus naturel. Gilbert Rist - tout comme, avant lui, Leimdorfer et al. (1986) -, s'interroge sur les dynamiques de légitimation de tels discours.

Notons aussi une exploration sémantico-argumentative approfondie du système logique du discours institutionnel européen sur l'espace judiciaire européen montrant que celui-ci est essentiellement un discours circulaire qui se nourrit à deux sources : l'obsession sécuritaire et le respect de la démocratie, sans cependant trancher entre les valeurs (Menuet, 2006). On peut rapprocher de ces approches sémantiques les travaux qui se réclament de Foucault et d'une méthode «généalogique » (Polzer, 2001) en ce qu'ils partagent, avec l'analyse critique du discours, une volonté non seulement analytique mais aussi critique. Ce que l'on retrouve dans l'étude de Jean-Louis Siroux (2008) sur les rapports annuels de l'OMC, où l'auteur, disséquant mots, figures de style et mobilisation d'indicateurs techniques, montre la dépolitisation apparente de ce discours qui pourtant impose l'universalité du libre-échange. Ce travail normatif avait déjà été repéré par Béatrice Hibou (1998) dans les discours de la Banque mondiale.

Les discours institutionnels sont des discours produits sous contrainte. Pour analyser les effets de ces contraintes sur l'information du message, il peut être utile d'associer, aux analyses lexicales ou argumentatives, une approche anthropologique et/ou organisationnelle. C'est le défi des travaux de Ruth Wodak et de ses collaborateurs (Muntigl et al., 2000), notamment dans la recherche collective portant sur les discours européens sur l'emploi et le nonemploi. L'observation ethnographique de la dynamique institutionnelle accompagne aussi l'analyse, par Michal Krzyżanowski et al. (2007), d'un ensemble 
de quarante entretiens des membres de la Convention européenne sur le futur de l'Europe, instituée en 2001 en vue de rédiger une Constitution européenne.

À partir de l'analyse des accords commerciaux, Rorden Wilkinson (2009) se penche sur l'usage d'une rhétorique spéciale - celle des «discours de crise»dans les négociations commerciales internationales.

Duchêne, quant à lui, a décortiqué la production d'un projet d'article du pacte économique et social à l'ONU et celle des comptes rendus des assemblées des Nations-Unies. Dans un cas, il met au jour la manière dont une question délicate (la protection des minorités) est, après conflit sur les termes, vidée peu à peu de sa substance; dans l'autre, il décrit les différentes transformations que subissent les paroles lors de leur transcription, les exigences contradictoires de concision et de fidélité obligeant le rédacteur à opérer des choix.

\section{Les analyses de contenu}

Relevons l'intérêt «militant» des organisations non gouvernementales pour la circulation de certains mots, notions ou concepts appartenant au champ sémantique de la mondialisation ou de la gouvernance dans les discours institutionnels. Cet intérêt trouve de plus en plus de relais dans des travaux universitaires. Ainsi a été analysée la mobilisation spécifique de pauvreté dans le discours de la Banque mondiale, dont la lutte contre la pauvreté, depuis 1990, est l'axe prioritaire d'action. Un parler expert qui aborde la pauvreté comme phénomène nécessairement multidimensionnel aboutit à relativiser l'importance des droits à rémunération (salaires, droits de sécurité sociale) : l'important n'est plus de changer de condition mais de vivre sa condition (Mestrum, 2008). Le «pauvre» devient une catégorie sociale positive (Corten, 1998). L'usage de la « responsabilité sociale» dans le discours de l'Organisation internationale du travail est exploré par l'historienne Chloé Maurel (2009), qui montre comment l'émergence de ce syntagme accompagne une modification profonde de l'attitude des agences de l'ONU envers les entreprises transnationales.

N'oublions pas non plus les travaux de type lexical qui tentent de rendre compte du sens spécifique des mots produits dans le contexte institutionnel spécifique à ces structures internationales (Bertoncini et al., 2008, Grossman et al., 2001).

Le dossier de Mots. Les langages du politique no 88 (Cussó et Gobin, 2008) abordait la question de la prolifération d'un «discours expert» au détriment du discours politique, produit par les institutions internationales dont l'Union européenne, et qui semblait se diffuser universellement (de façon transgéographique et transpartisan), occultant en quelque sorte les tensions et les conflits inhérents à la vie politique démocratique. Cette analyse «macrosociale» ne 
doit cependant nullement dispenser de l'important travail scientifique portant sur la nuance et la réappropriation de ce discours expert par de multiples acteurs. C'est pourquoi le thème de la «circulation lexicale» est important aujourd'hui. Non seulement pour repérer le lieu originel de création de ces « formules » (Krieg-Planque, 2009) technocratiques, inlassablement répétées, mais surtout pour voir ce qu'en fait le reste de la production politique.

Un autre chantier passionnant mais peu traité (Deroubaix, 2004) : la collecte de corpus de textes d'institutions internationales dans leurs multiples versions linguistiques pour une analyse fine des variations dues à la retranscription d'un message dans un univers sémantico-linguistique particulier. Rien ne prouve que l'Union européenne s'adressant à ses «citoyens » dans vingt-trois langues officielles parle bien, à chaque fois, de la même chose...

\section{Références}

BERTONCINI Y. et al., 2008, Dictionnaire critique de l'Union européenne, Paris, Armand Colin.

CORTEN A., 1998, "Le discours de la pauvreté de la Banque mondiale», Langage et société, n०85, p. 5-24.

Cussó R., 2008, «Quand la Commission européenne promeut la société de la connaissance ", Mots. Les langages du politique, n88, novembre, p. 39-52.

Cussó R., Gobın C., 2008, «Du discours politique au discours expert. Le changement politique mis hors débat?", Mots. Les langages du politique, n88, novembre, p. 5-11.

Deroubaix J.-C., 1995, «La périodisation des corpus de discours politiques. Comparaison de deux corpus de discours de présentation : les gouvernements belges et la Commission européenne», $3^{\mathrm{e}}$ JADT, Rome, 11-13 décembre 1995, 2 vol., S. Bolasco, L. Lebart, A. Salem éd., Rome, CISU, p. 201-209.

- 2004, "Que faire des corpus multilingues parallèles? Une expérience», Le poids des mots, $7^{\mathrm{e}}$ JADT, vol.l, Louvain-la-Neuve, Presses universitaires de Louvain, p. 295-303.

Deroubaix J.-C., Gobin C., 1994, Quand la Commission se présente devant le Parlement. Étude du vocabulaire des discours de présentation de la Haute Autorité de la CECA et de la Commission de la Communauté européenne devant le Parlement européen (1952-1993), rapport édité par l'ASBL RESH pour la Commission européenne, DG X, Bruxelles, vol.I, en ligne [URL : http://difusion.ulb.ac.be/vufind/Record/ULBDIPOT:oai:dipot.ulb.ac.be:2013/49933/Holdings]. Site consulté le 13 juillet 2010.

DUCHÊNEA., 2004, «Construction institutionnelle des discours. Idéologies et pratiques dans une organisation supranationale», Travaux neuchâtelois de linguistique, no 40 , p. $93-115$.

- 2006, " “Dans les États où il existe des minorités..." Les conditions de production 
institutionnelle, discursive et idéologique d'un article de loi aux Nations-Unies », Semen, $\mathrm{n}^{\circ} 21, \mathrm{p} .121-140$.

DUfRESNE A., 2004, "Le discours de la Banque centrale européenne concernant les aspects sociaux. Le poids des mots ", $7^{\mathrm{e}}$ JADT, vol. I, Louvain-la-Neuve, Presses universitaires de Louvain, p. 373-381.

Fairclough N., 2005, "Critical discourse analysis», Marges linguistiques, n9, p. 76-94.

Gobin C., 1993, «La Confédération européenne des syndicats. Un vocabulaire syndical européen?", Mots. Les langages du politique, n³ 36 , septembre, p. 33-47.

- 2000, "Un survol des discours de présentation de l'exécutif européen (19581993)", Mots. Les langages du politique, no62, mars, p. 99-107.

- 2003, "De l'international au mondial. La CES aux prises avec la mondialisation», Mots. Les langages du politique, $\mathrm{n}^{0} 71$, mars, p. 67-84.

- 2005, «L'Union européenne. Où est passé l'acteur?», Cahiers lillois d'économie et de sociologie, $\mathrm{n}^{\circ} 45$, Action et domination dans les relations de travail, Paris, L'Harmattan, p. 65-88.

Gobin C., Deroubaix J.-C., 2009, «Mots, fréquence et réseaux dans le discours politique. Analyse lexicométrique, méthode et illustration dans deux corpus de textes européens », Sociolinguistic Studies, vol. III, n² 2, p. 203-227.

Gobin C. éd. et al., 2005, Glossaire des discours européens sur les réformes des pensions et de l'emploi, Deliverable n³3, Recherche européenne RESORE.

Grossman E. et al., 2001, Les mots de l'Europe. Lexique de l'intégration européenne, Paris, Presses de Sciences Po.

Hıвоu B., 1998, "Économie politique du discours de la Banque mondiale en Afrique sub-saharienne», Les études du CERI, nº39, en ligne [URL : http://www.cerisciencespo.com/publica/etude/etude39.pdf]. Site consulté le 13 juillet 2010.

KRIEg-PlAnque A., 2009, La notion de "formule» en analyse du discours. Cadre théorique et méthodologique, Besançon, Presses universitaires de Franche-Comté.

KrzyŻAnowskı M., Oberhuber F., 2007, (Un)doing Europe. Discourses and Pratices of Negociating the EU Constitution, Bruxelles, Peter Lang.

Leimdorfer F., TeSsonneau A.-L., 1986, "Légitimité sociologique et analyse de discours. Les parcours de la légitimité dans les textes de l'Unesco sur l'éducation de base», Langage et société, n³ 37, p. 61-111.

MAUREL C., 2009, "OIT et responsabilités sociales des entreprises transnationales depuis les années 1970 ", Communication pour le colloque Politiques sociales transnationales. Réseaux réformateurs et Organisation internationale du travail (1900-1980), en ligne [URL: http://www.unige.ch/ieug/recherche/colloques/ILO/ Maurel.pdf]. Site consulté le 13 juillet 2010.

Menuet L., 2006, «Le discours sur l'espace judiciaire européen. Analyse du discours et sémantique argumentative», thèse de doctorat, Université libre de Bruxelles et Université de Nantes.

Mestrum F., 2008, "La pauvreté multidimensionnelle. La dynamique sémantique dans le discours de la Banque mondiale ", Mots. Les langages du politique, n 88 , novembre, p. 25-38. 
MUNTIGL P., Weiss G., WodAK R., 2000, «European Union discourses on un/employment. An interdisciplinary approach to employment policy-making and organizational change », Dialogues on Work and Innovation, $\mathrm{n}^{\circ} 12$.

Polzer T., 2001, "Corruption. Deconstructing the World Bank Discourse», Working Paper Serie, $n^{\circ} 1$ (18), Londres, Development Studies Institute, London School of Economics.

Proulx Y., 2001, "La transformation des politiques sociales destinées aux sansemploi. Une analyse du discours de l'OCDE (1975-1999)», Mémoire, UQUAM, en ligne [URL: http://hussonet.free.fr/proulx.pdf]. Site consulté le 13 juillet 2010.

RIST G. éd., 2002, Les mots du pouvoir. Sens et non-sens de la rhétorique internationale, Paris, PUF (Nouveaux cahiers de l'IUED).

ROBERTSON S., 2005, "Re-imagining and rescripting the future of education : global knowledge economy discourses and the challenge to education systems », Comparative Education, vol. XLI, nº 2, p.151-170.

Siroux J.-L., 2008, "La dépolitisation du discours au sein des rapports annuels de l'Organisation mondiale du commerce", Mots. Les langages du politique, n 88 , novembre, p.13-23.

WAGNER A.-C., 2008, «L'expertise syndicale européenne. L'imbrication des lexiques de l'expertise et du discours militant chez les syndicalistes européens », Mots. Les langages du politique, n०88, novembre, p. 53-65.

WILKINSON R., 2009, "Language, power and multilateral trade negotiations », Review of International Political Economy, vol.XVI, $\mathrm{n}^{\circ}$ 4, p.597-619.

Wodak R., Chilton P. éd., 2005, A New Agenda in (Critical) Discourse Analysis, Amsterdam, Philadelphia, Benjamins. 